\title{
Bioassay-guided evaluation of wound healing effect of fatty acids-incorporated collagen-based films ${ }^{1}$
}

\author{
Catharina Grace Santos', Marismar Fernandes do Nascimento ${ }^{\mathrm{I}}$, Clauberto Rodrigues de Oliveira ${ }^{\mathrm{II}}$, Genecy Calado de Melo ${ }^{\mathrm{III}}$, \\ Juliana Cordeiro Cardoso ${ }^{\mathrm{IV}}$, Francine Ferreira Padilha ${ }^{\mathrm{Iv}}$, Sonia Oliveira Limav, Ricardo Luiz Cavalcanti de Albuquerque- \\ Júnior ${ }^{\mathrm{VI}}$ \\ ${ }^{\mathrm{I}}$ Fellow Master degree, Postgraduate Program in Health and Environment, University Tiradentes, Aracaju-SE, Brazil. Technical procedures, manuscript \\ writing. \\ IFellow Master degree, Postgraduate Program in Industrial Biotechnology, University Tiradentes, Aracaju-SE, Brazil. Technical procedures of films \\ manufacturing, statistical analysis. \\ IIIGraduate student, Department of Dentistry, University Tiradentes, Aracaju-SE, Brazil. Involved with histological processing of the surgical samples. \\ ${ }^{\text {IV }} \mathrm{PhD}$, Full Professor, Laboratory of Biomaterials, Department of Pharmacy, University Tiradentes, Aracaju-SE, Brazil. Manuscript writing, critical \\ revision. \\ vPhD, Full Professor, Department of Medicine, University Tiradentes, Aracaju-SE, Brazil. Supervision of the surgical procedures in rodent model.

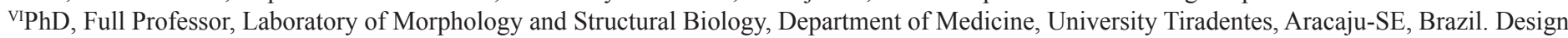 \\ of the study, histological examinations, manuscript writing, supervised all phases of the study.
}

\begin{abstract}
PURPOSE: To evaluate the effects of fatty acids-incorporated collagen-based dressing films on wound healing in rodents.

METHODS: Therefore, surgical wounds were performed in the back of 80 Wistar rats, and dressed with collgane-based films (COL), and collagen-based films containing fatty acids (AGEF50 and AGEF100). Undressed wounds were regarded as controls (CTR). The animals were euthanized after three, seven, 14 and 21 days, and the macroscopic wound contraction rates (WRC) were assessed. The wounded area was also analyzed by conventional and polarized light microscope.

RESULTS: No sign of abscess or hypertrophic scar formation was observed in none of the groups. At seven days, the WRR of AGEF50 was significantly higher than CTR ( $<<0.01$ ), whereas at 14 days, both AGE 50 and AGE100 showed a significant increase of the WRR compared to CTR $(p<0.001)$ and COL $(p<0.01)$. Both films promoted increased influx of neutrophils at three days ( $<<0.01)$, but reduced significantly the mononuclear infiltrate at 14 days $(\mathrm{p}<0.05)$. It was also observed earlier maturation of the granulation tissue, full epithelization and cutaneous appendages development, as well as better collagenization, in AGEF50 and AGEF100.
\end{abstract}

CONCLUSION: The application of AGEF50/100 as wound dressing improved wound healing in rodents.

Key words: Wound Healing. Fatty Acids. Biofilms. Rats. 


\section{Introduction}

Wound healing is a complex process that occurs in three overlapping pathophysiological phases named inflammatory reaction, proliferation, and remodeling ${ }^{1}$. The inflammatory phase is characterized by vascular events which allow the influx of leukocytes into the wound site, responsible for microbial and necrotic tissue elimination ${ }^{2}$. The proliferative phase refers to the restoration of the epidermis and collagen formation, and involves migration and proliferation of keratinocytes from the wound edges, as well as fibroblast and endothelial proliferation to form the granulation tissue and further primary collagen scar. The remodeling phase starts during the proliferative phase but continues for a long time after injury (up to many months), and is characterized by increased collagen turnover and wound contraction in an attempt to restore skin morphological and functional integrity ${ }^{3}$.

However, in the case of full-thickness burn injuries and chronic wounds such as pressure ulcers, venous ulcers and diabetic foot ulcers these processes are damaged and new technologies have been developed to improve the healing in these conditions ${ }^{4}$. Thus, the last two decades have witnessed the introduction of many new dressings, based on the concept of creating an optimum environment to allow epithelial cells to move unimpeded, for the treatment of wounds ${ }^{5}$. Such optimum conditions include a moist environment around the wound, effective oxygen circulation to aid regenerating cells and tissues and a low bacterial load ${ }^{6}$.

Collagen matrix is known to provide physical support for cellular proliferation and qualifies as an excellent material for wound dressing due to its biodegradable and biocompatible properties ${ }^{7}$. In addition, the incorporation of bioactive molecules, such as curcumin ${ }^{8}$ and usnic acid ${ }^{9}$, or natural products, such as red propolis extract ${ }^{10}$, into collagen-based films have been successfully used to improve wound healing in experimental models.

Fatty acids are chemical compounds that present a long hydrocarbonate chain and a carboxyl-terminus group, which compose the biological membrane structures; function as precursors of intracellular messengers; and are oxidizers, generating adenosine triphosphate (ATP) and have been applied to wound treatment ${ }^{11}$. Studies have demonstrated that fatty acids are able to modulate a wide range of biological activities involved in wound healing, such as differentiation of fibroblasts into myofibroblasts ${ }^{12}$, fibroblasts proliferation ${ }^{13}$, endothelial-leukocyte adhesion ${ }^{14}$. Keratinocytes differentiation and development of the cutaneous appendages ${ }^{15}$.

Hence, this study aimed to evaluate the healing potential of fatty acids-incorporated collagen-based films on secondintention wound healing in rodent model.

\section{Methods}

The animals used in this study were adult male Rattus norvegicus albinus, Wistar lineage, weighing 250-300g. The rats were housed in clear plastic cages with solid floors and loose hardwood chip bedding, and supplied with food and water ad libitum in a temperature and humidity-controlled environment. Experimental protocols and procedures were approved by the University Tiradentes Animal Care and Use Committee (CEUA $\left.n^{\circ} 440911\right)$.

\section{Surgical procedures and groups}

Eighty rats were anesthetized with intraperitoneal ketamine-xylazine $(100 \mathrm{mg} / \mathrm{kg}-5 \mathrm{mg} / \mathrm{kg})$ and standard-sized round-shaped surgical wounds were performed in the back of the animals, using metallic punch of $8.0 \mathrm{~mm}$. Animals were handled in accordance to the principles of aseptic chain in order to avoid bacterial contamination. Subsequently, rats were randomly assigned into four groups of 20 animals each: CTR, undressed wounds; COL, dressed with collagen-based films; AGEF50 - dressed collagen-based films containing AGE50 ${ }^{\circledR}$ fatty acids formulation; and AGEF100 - dressed collagen-based films containing AGE100 ${ }^{\circledR}$ fatty acids formulation. After three, seven, 14 and 21 days, five animals of each group were euthanized in $\mathrm{CO}_{2}$ chamber, and the healing/scar area was surgically removed, formalin-fixed and paraffin-embedded.

For this study, it was used gelatin (Gellita South America, Cotia/SP, Brazil), glycerol, tween 20 and 80, acetic acid (Vetec Quimica Fina Ltda, Rio de Janeiro/RJ, Brazil), commercial formulation of essential fatty acids containing oleic/linoleic acids (AGE100 ${ }^{\circledR} /$ Tecpon, Cachoeirinha/RS, Brazil), commercial formulation of essential fatty acids with oleic/linoleic acids and sunflower oil (AGE50 ${ }^{\circledR} /$ Tecpon, Cachoeirinha/RS, Brazil).

Films preparation

The films were prepares by casting method from a solutions of $2.13 \%$ of gelatin powder solubilised in acetic acid $(0.5 \mathrm{M})$ in a vessel equipped with a mechanical stirrer, with heating to $55^{\circ} \mathrm{C}$ for 20 minutes. Glycerin $(15 \%$ or $20 \% \mathrm{w} / \mathrm{w})$ as plasticizer and tween 20 or $80(9.4 \%$ or $4.7 \% \mathrm{w} / \mathrm{w})$ as emulsifier, respectively, were added to solution while stirring. Thereafter, fatty acid AGE 50 or AGE $100(10 \% \mathrm{w} / \mathrm{w}$ based on the weight of the gelatin powder) (TECPON, Brazil) added to the solutions, the 
compositions of the formulations films are listed in Table 1. The solution was homogenized in an Alpax Ltda, Diadema/SP, Brazil) at $30.000 \mathrm{rpm}$ for $10 \mathrm{~min}$. The emulsion $(100 \mathrm{~mL})$ was poured into disposable Petri dish, placed on a leveled surface, and dried at $40^{\circ} \mathrm{C}$ for $24 \mathrm{~h}$. The obtained films were peeled from the disposable Petri dish plates and stored at $25^{\circ} \mathrm{C}$ for $48 \mathrm{~h}$ in desiccators at $58 \%$ relative humidity $(\mathrm{RH})$ before the tests were carried out. The prepared films with a size of $2 \times 3 \mathrm{~cm}^{2}$ were sterilized for $30 \mathrm{~min}$ in UV-irradiation before use.

TABLE 1 - Compositions of the formulations films.

\begin{tabular}{ccc}
\hline Experimental Film & Glycerol & Solvent \\
\hline AGEF 50 & $15 \%$ & Tween $80(4.7 \%)$ \\
AGEF 100 & $20 \%$ & Tween $20(9.4 \%)$ \\
\hline
\end{tabular}

\section{Assessment of the wound contraction rates}

For the macroscopic analysis and assessment of the wound contraction rates, after three, seven, 14, and 21 day, the craniocaudal and latero-lateral measures of each wound were assessed by a digital caliper (precision $0.01 \mathrm{~mm}$ ), prior to the excision of the wounds, and the final wound areas were obtained through the equation $\mathrm{A}=\pi \times \mathrm{R} \times \mathrm{r}$, where $\mathrm{R}$ represents the craniocaudal axis and $\mathrm{r}$ corresponds to the latero-lateral axis of the wounds. Clinical features of the wounds were monitored regarding to the presence of crust, secretion, necrosis, and hypertrophic scars.

\section{Histological procedures and morphological analysis}

Serial $5 \mu \mathrm{m}$ thick sections were obtained from the paraffin-embedded samples and stained in hematoxylin-eosin. The intensity of the inflammatory response was assessed as follows: 0 - absence of inflammatory infiltrate; 1 - Inflammatory cells representing less than $10 \%$ of the cell population; 2 inflammatory cells representing between $10 \%$ and $50 \%$ of the cell population; and 3 - inflammatory cells representing more than $50 \%$ of the cell population. The inflammatory profile was classified as acute (predominance of polymorphonuclear cells) and chronic (predominance of mononuclear cells). For the assessment of the collagen deposition, histological sections stained in Sirius Red and analyzed under polarized light were used to the descriptive analysis. Collagen fibers were analyzed according their birefringence pattern (greenish/yellow-greenish or orange, orange-reddish), morphological appearance (wavy or stretched, thin or thick, short or long), and architectural arrangement (reticular, parallel or interlaced). All readings were performed by investigators blinded to treatments (six histological sections/animal).

\section{Statistical analysis}

The data concerning the wound contraction rates were analyzed using ANOVA followed by Tukey's test, whereas the analysis of the inflammatory infiltrate was carried out by KruskalWallis test, followed by Dunn's test. P values less than 0.05 were considered significant.

\section{Results}

Macroscopic analysis and wound contraction rates assessment

Figure 1 presents the macroscopic aspects of the wounds over the time course of the experiment, showing no sign of abscess formation in the early phases (three and seven days) or hypertrophic cars in the final ones (14 and 21 days).

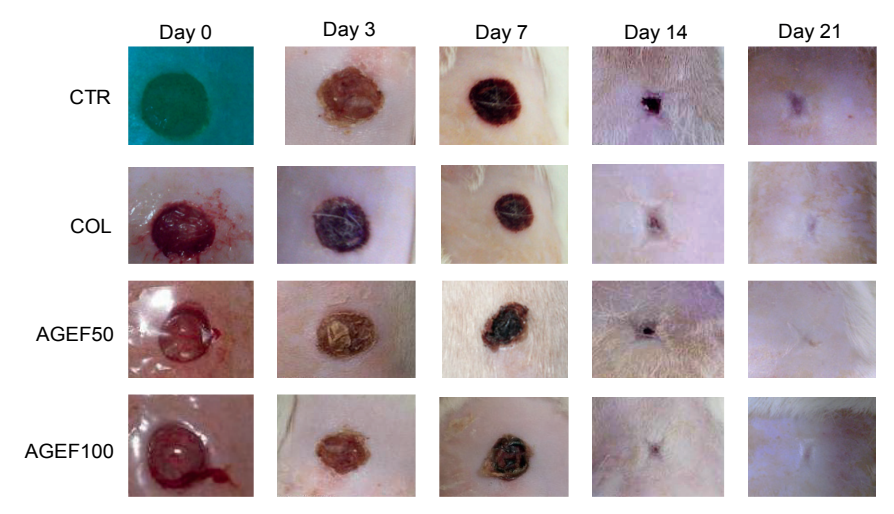

FIGURE 1 - Macroscopic features of the wounds of the different groups over the time-course of the experiment.

As demonstrated in Figure 2, no significant difference was observed in the WRR of the groups in three days ( $>0.05)$. In seven days, the WRR of AGEF50 was significantly higher than CTR ( $p<0.01)$, whereas in 14 days, both AGE 50 and AGEF100 showed a significant increase of the WRR compared to CTR $(p<0.001$ and 0.001$)$ and COL (0.001 and 0.01). In 21 days, all the groups presented full retraction of the wounds. 


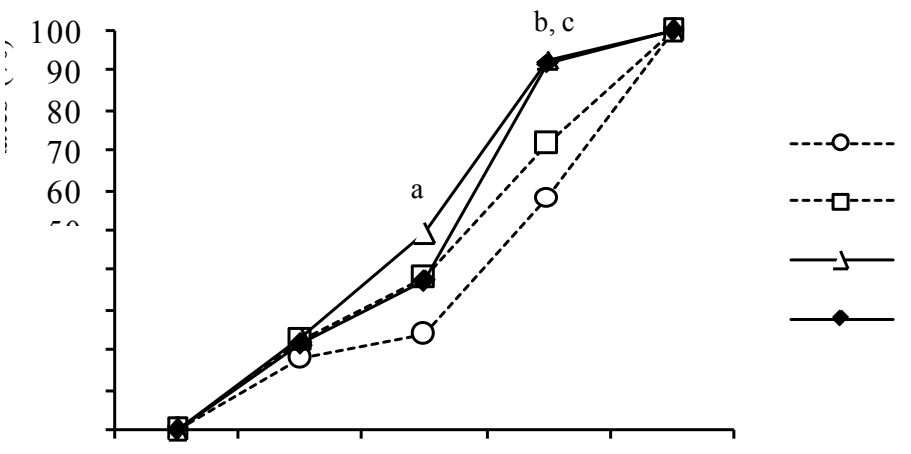

FIGURE 2 - Assessment of the wound retraction rates (WRR) throughout the time.
(a) AGE50 is significantly different from CTR ( $<<0.01)$.
(b) AGE50 is significantly different from CTR $(\mathrm{p}<0.001)$ and COL $(\mathrm{p}<0.001)$.

(c) AGE100 is significantly different from CTR $(\mathrm{p}<0.001)$ and $\operatorname{COL}(\mathrm{p}<0.01)$.

\section{Histological analysis}

In three days, all the groups presented intense plasmatic exudation, associated to marked infiltration of polymorphonuclear neutrophils. Interstitial edema was remarkable in the bottom of the wounds, whereas mild vascular neoformation was evidenced in the marginal wounded areas. In seven days, CTR, COL and AGEF100 exhibited immature granulation tissue, with formation of numerous irregular capillary vessels and intense infiltration of lymphocytes and histiocytes. In AGEF50, the granulation tissue appeared more mature, with remarkable fibroblastic proliferation as well as deposition of parallel-arranged collagen fibrils. The epithelization process recovered less than $30 \%$ of the wounded area in all the groups. In 14 days, the epithelial lining recovered more than $70 \%$ of the wound surface in CTR and COL, but only two samples of both groups presented full epithelization. However, the epithelization process was complete in $100 \%$ of AGEF50 and AGEF100. Moreover, rudiments of cutaneous appendages were observed budding from the epithelium line, particularly I the margins of the wounds. The primary scar was well developed, with intense fibroblastic proliferation, and mild residual granulation tissue. In 21 days, there was full epithelization in all the groups, although the cutaneous appendages were only observed in AGEF50 and AGEF100. The connective tissue was intensely fibrotic, with absence of inflammatory infiltrate in all the groups (Figure 3).

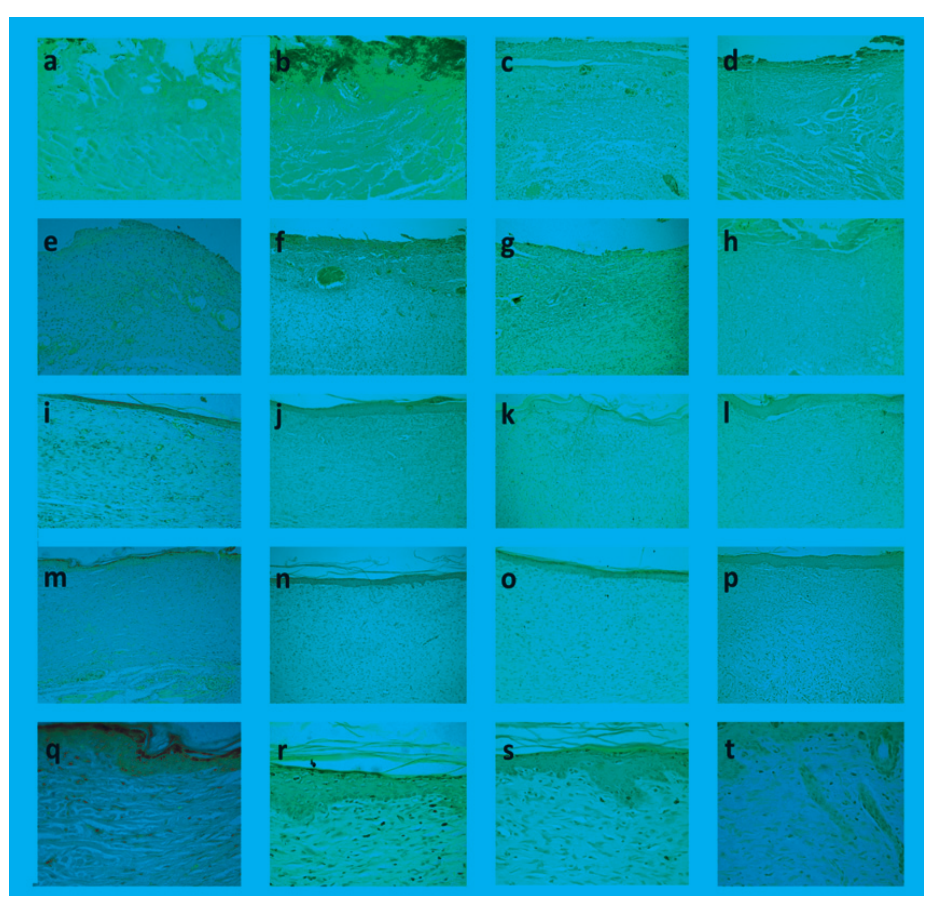

FIGURE 3 - Histological findings observed in CTR (a/e/i/m/q), COL (b/f/ $\mathrm{j} / \mathrm{n} / \mathrm{r}), \operatorname{AGEF} 50(\mathrm{c} / \mathrm{g} / \mathrm{k} / \mathrm{o} / \mathrm{s})$ and $\operatorname{AGEF} 100(\mathrm{~d} / \mathrm{h} / \mathrm{l} / \mathrm{p} / \mathrm{t})$. (a-d) inflammatory phase of wound healing at three days, (e-h) granulation tissue formation at seven days, (i-l) development of the primary fibrous scar at 14 days, (h-p) well developed fibrous scar at 21 days in the experimental groups (H\&E, 100x), (q-t) details of the epithelial features, showing formation of cutaneous appenfages only in AGEF50 and AGEF100 (400x).

As shown in Figure 4, the intensity of the inflammatory infiltrate decrease over the time course of the experiment. At three days, AGEF50 and AGEF100 presented increased polymorphonuclear neutrophils infiltrate compared to CTR $(\mathrm{p}<0.01)$, whereas in 14 days, the mononuclear inflammatory content was significantly reduced in both groups $(\mathrm{p}<0.05)$. No difference among the groups was observed in the inflammatory cells content in seven and 21 days $(p>0.05)$. 

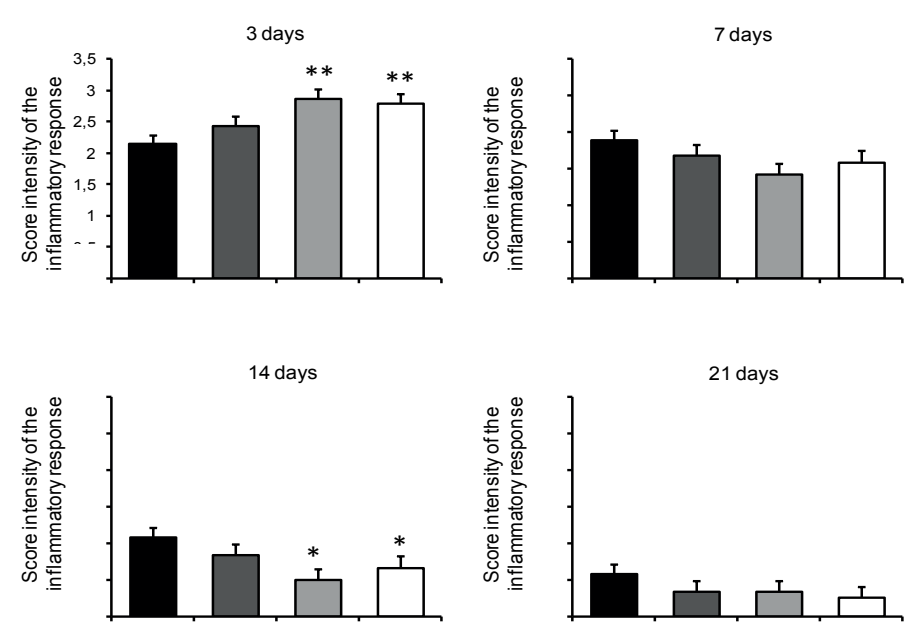

FIGURE 4 - Histological assessment of the mean scores $( \pm \mathrm{SD})$ of the intensity of the inflammatpry response in the studied groups over the time course of the experiment.

*Significantly different from CTR $(\mathrm{p}<0.05)$.

**Significantly different from CTR $(\mathrm{p}<0.01)$.

\section{Morphological analysis of collagenization}

In three days, extremely delicate greenish or yellowgreenish fibrillar structures (type-III collagen) with varied dimensions and reticular dispositions were observed in all the groups. In seven days, type-III collagen fibers thicker and denser, disposed in a reticular arrangement in CTR, and COL, and parallel arranged in AGE50 and AGE100. In 14 days, there was replacement of type III for type I collagen fibers, which were short, thin, delicate and irregularly disposed in CTR but longer and arranged in a parallel disposition in COL, AGE50 and AGE100 (Figure 5).

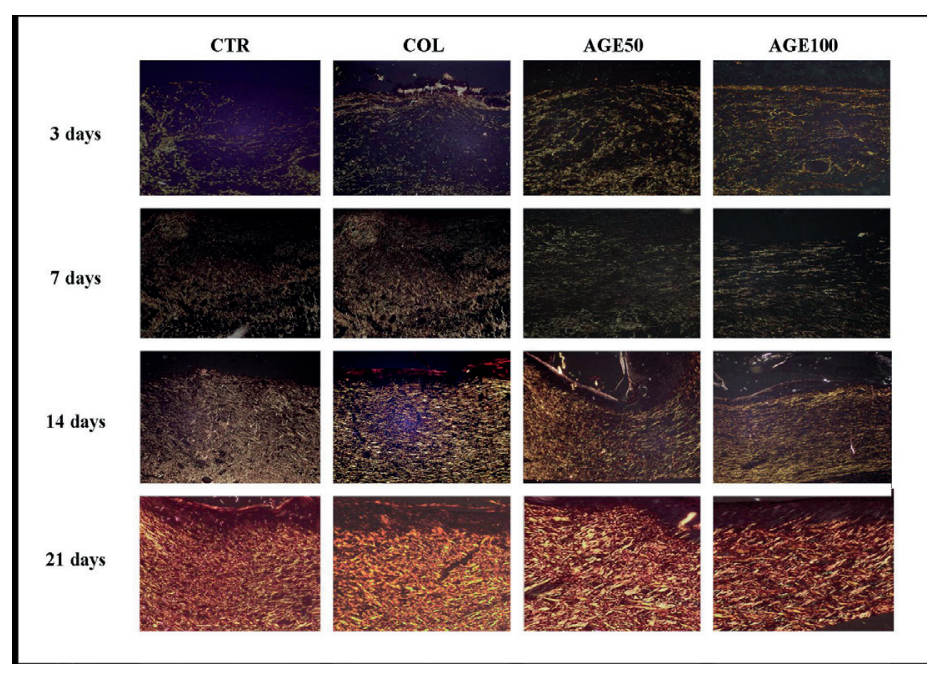

FIGURE 5 - Collagen deposition in the experimental groups during the time-course of the study. In three and seven days, there was a predominance of thin delicate type III collagen fibrils (greenish birefringence), whereas in 14 these fibrils were replaced by thicker and longer type I collagen fibers. In 21 days, all the groups presented grossly interlaced type I collagen fibers (Sirius Red/Polarized Light, 400x).

\section{Discussion}

The increased wound contraction rates observed in the wounds dressed with AGEF50 and AGEF100 suggest that the presence of essential fatty acids improved significantly the healing process. It is well established that the wound contraction is closely associated to the differentiation fibroblasts into myofibroblasts, a phenotypic change mediated by transforming growth factor beta (TGF- $\beta$ ), a cytokine widely released by mononuclear inflammatory cells within the wound ${ }^{16}$. Once it has been previously reported that fatty acids are able to enhance the synthesis and release of TGF- $\beta$, and induce the expression of contractile antigens ( $\alpha$ smooth muscle actin) in fibroblasts, changing them into a myofibroblastic phenotype, in experimental in vitro studies ${ }^{12}$, it is possible to speculate that such increased contraction rates might be likely related to higher levels of TGF- $\beta$ and more expressive myofibroblastic differentiation. Therefore, the lack of significant difference among the groups at three days seems to support this theory, since the inflammatory response was rich in polymorphonuclear instead of mononuclear cells, which are the main source of TGF- $\beta$. Moreover, at 21 days, a substantial apoptosis-mediated decrease of the myofibroblats is expected ${ }^{17}$, which justifies the findings obtained in this study. Nevertheless, further investigations analyzing the variations in the myofibroblasts subsets by immunohistochemical and molecular methods are necessary in order to assess the validity of such hypothesis.

The morphological evidence of a mature more cellular 
and less vascular granulation tissue observed in AGEF50 at seven days suggest an acceleration of the biological events of wound healing. It has been recently reported that oleic and linoleic acids induce fibroblasts proliferation in vitro ${ }^{13}$. Therefore, the high content of such fatty acids in AGE $50^{\circledR}$ formulation might be related with the improvement in the fibroblast-consistent spindle-shaped cell component, promoting earlier maturation of the granulation tissue. Moreover, unsaturated fatty acids, such as oleic acid, seem to exert anti-inflammatory activity by modulating the endothelialleukocyte adhesion ${ }^{14}$. This biological property might shorten the acute phase of the inflammatory response, and consequently accelerate the installation and development of the granulation tissue.

The semi-quantitative analysis of the intensity of the inflammatory response showed that both fatty acids-incorporated collagen-based films increased significantly the influx of neutrophils into the wounded area during the acute inflammatory phase of wound healing. Similar findings have been previously reported $^{18}$, who also demonstrated that such increase in the polymorphonuclear content content were likely related to an oleic and linoleic-induced enhancement of the expression of vascular endothelial growth factor-alpha (VEGF-alpha) by neutrophils, improving the endothelial-leukocyte adhesion, as well as an increased release of cytokine-induced neutrophil chemoattractant 2 alpha/beta (CINC-2alpha/beta), a potent chemotactic cytokine during the inflammatory response. Nevertheless, it has been recently reported that oleic acid is able to downregulate longterm inflammatory responses ${ }^{19}$, and suggested that this biological phenomenon might be related to apoptosis of $\mathrm{T}$ cells mediated by oleic acid exposure. The reduced content of monulcear inflammatory cells in AGEF50 and AGEF100 observed at 14 days seems to support this theory. Therefore, it is possible to suggest that the presence of fatty acids might have speeded-up the timecourse of the inflammatory response, by accelerating the acute phase and shortening the chronic phase.

The analysis of the epithelization process in 14 days suggested that the fatty acids might have played a modulatory role on the proliferation and migration of epidermal keratinocytes, accelerating the full epithelial recovery of the wound surface. In fact, it has been previously reported that keratinocytes use fatty acids to synthesize lamellar bodies, which are regarded as important structures to the formation of the cell lipid membranes ${ }^{20}$. In addition, the experimental inhibition of fatty acids blocks the formation of such lamellar bodies, impairing the keratinization process and maturation of the epidermal tissue ${ }^{15}$.

The earlier development of dermal appendages was also observed in AGEF 50 and AGEF 100. Recent essays have demonstrated the over expression of fatty acids transport proteins (FATP) over the time-course of the development of the epidermis and cutaneous appendages, suggesting the active participation of such lipid molecules in the formation and maturation of the epidermal-derived structures ${ }^{15}$. Accordingly, it is possible to suggest that the additional availability of fatty acids, derived from AGEF50 and AGEF100, might have increased the keratinocytes metabolism, resulting in earlier formation of mature keratinized epithelial lining and cutaneous appendages. However, further research assessing the FATP expression in epithelial tissues during wound healing is necessary to investigate the precise mechanisms underlying the keratinocytes metabolic enhancement in response to the increased fatty acids supply.

The analysis of the collagenization seemed to corroborate the hypothesis of a possible stimulatory effect of fatty acids on the fibroblasts, as previously reported by others studies ${ }^{13}$. Notwithstanding, the modulatory effects observed in this study appeared to be related to an improvement of both synthesis and degradation of the collagen molecules, promoting faster remodeling of the collagen scar, without excessive deposition of these fibers, which would ultimately lead to hypertrophic scar formation.

\section{Conclusion}

The application of AGEF50/100 as wound dressing improved wound healing in rats and presented better results than AGEF100.

\section{References}

1. Broughton G 2nd, Janis JE, Attinger CE. Wound healing: an overview. Plast Reconstr Surg. 2006;117(7 Suppl):1e-S-32e-S

2. Li J, Chen J, Kisher R. Pathophysiology of acute wound healing. Clin Dermatol. 2007;25:9-18.

3. Rangaraj A, Harding K, Leaper D. Role of collagen in wound management. Wounds. 2011;7(2):54-63.

4. Weller C, Sussman G. Wound dressings update. J Pharm Pract Res. 2006;36(4):318-24.

5. Boateng JS, Matthews KH, Stevens HN, Eccleston GM. Wound healing dressings and drug delivery systems: a review. J Pharm Sci. 2008;97(8):2892-923.

6. Murphy PS, Evans GR. Advances in wound healing: a review of current wound healing products. Plast Surg Int. 2012;2012:190436.

7. Deyl Z, Adam M. Preparation of insoluble collagen. In: Hall DA, editor. The methodology of connective tissue research. 1ed. Oxford: Joynson-Bruvvers; 1976. p.1-7.

8. Gopinath D, Ahmed MR, Gomathi K, Chitra K, Sehgal PK, Jayakumar R. Dermal wound healing processes with curcumin incorporated collagen films. Biomaterials. 2004;25(10):1911-7. 
9. Nunes PS, Albuquerque RL Jr, Cavalcante DR, Dantas MD, Cardoso JC, Bezerra MS, Souza JC, Serafini MR, Quitans LJ Jr, Bonjardim LR, Araújo AA. Collagen-based films containing liposome-loaded usnic acid as dressing for dermal burn healing. J Biomed Biotechnol. 2011;2011:761593.

10. Albuquerque-Júnior RL, Barreto AL, Pires JA, Reis FP, Lima SO, Ribeiro MA, Cardoso JC. Effect of bovine type-I collagen-based films containing red propolis on dermal wound healing in rodent model. Int J Morphol. 2009;27(4):1105-10.

11. Ferreira AM, de Souza BM, Rigotti MA, Loureiro MR. The use of fatty acids in wound care: an integrative review of the Brazilian literature. Rev Esc Enferm USP. 2012;46(3):752-60.

12. Mishra R, Simonson MS. Oleate induces a myofibroblast-like phenotype in mesangial cells. Arterioscler Thromb Vasc Biol. 2008;28(3):541-7.

13. Magdalon J, Hatanaka E, Romanatto T, Rodrigues HG, Kuwabara WM, Scaife C, Newsholme P, Curi R. A proteomic analysis of the functional effects of fatty acids in NIH $3 \mathrm{~T} 3$ fibroblasts. Lipids Health Dis. 2011;10:218-26.

14. Ibrahim A, Mbodji K, Hassan A, Aziz M, Boukhettala N, Coëffier M, Savoye G, Déchelotte P, Marion-Letellier R. Anti-inflammatory and anti-angiogenic effect of long chain n-3 polyunsaturated fatty acids in intestinal microvascular endothelium. Clin Nutr. 2011;30(5):67887.

15. Schmuth M, Neyer S, Rainer C, Grassegger A, Fritsch P, Romani N, Heufler C. Expression of the C-C chemokine MIP-3 alpha/CCL20 in human epidermis with impaired permeability barrier function. Exp Dermatol. 2002;11(2):135-42.

16. Thannickal VJ, Lee DY, White ES, Cui Z, Larios JM, Chacon R, Horowitz JC, Day RM, Thomas PE. Myofibroblast differentiation by transforming growth factor-betal is dependent on cell adhesion and integrin signaling via focal adhesion kinase. J Biol Chem. 2003;278(14):12384-9.

17. Ribeiro MA, Albuquerque RL Jr, Ramalho LM, Pinheiro AL, Bonjardim LR, Da Cunha SS. Immunohistochemical assessment of myofibroblasts and lymphoid cells during wound healing in rats subjected to laser photobiomodulation at $660 \mathrm{~nm}$. Photomed Laser Surg. 2009;27(1):49-55.

18. Pereira LM, Hatanaka E, Martins EF, Oliveira F, Liberti EA, Farsky $\mathrm{SH}$, Curi R, Pithon-Curi TC. Effect of oleic and linoleic acids on the inflammatory phase of wound healing in rats. Cell Biochem Funct. 2008;26(2):197-204.

19. Carrillo C, Cavia M, Alonso-Torre S. Role of oleic acid in immune system; mechanism of action: a review. Nutr Hosp. 2012;27(4):97890

20. Feingold KR. The regulation and role of epidermal lipid synthesis. Adv Lipid Res. 1991;24:57-82.

\section{Correspondence:}

Ricardo Luiz Cavalcanti de Albuquerque-Júnior

Laboratório de Morfologia e Biologia Estrutural

Instituto de Tecnologia e Pesquisa, Universidade Tiradentes

Avenida Murilo Dantas, 300,

49032-490 Aracaju-SE Brasil

Tel.: (55 79)3218-2190, ramal 2615

ricardo.patologia@uol.com.br

Received: January 15, 2013

Review: March 19, 2013

Accepted: April 17, 2013

Conflict of interest: none

Financial source: Foundation for Research and Technological Innovation Support from Sergipe (FAPITEC/SE)

${ }^{1}$ Research performed at Laboratory of Morphology and Structural Biology (LMBE), ITP (Science and Technology Institute), University Tiradentes, Aracaju-SE, Brazil. Part of Master degree thesis, Postgraduate Program in Health and Environment. Tutor: Prof. Dr. Ricardo Luiz Cavalcanti de Albuquerque Júnior. 